\title{
Haptic pop-out of movable stimuli
}

\author{
Vonne van Polanen • Wouter M. Bergmann Tiest • \\ Astrid M. L. Kappers
}

Published online: 18 October 2011

(C) The Author(s) 2011. This article is published with open access at Springerlink.com

\begin{abstract}
When, in visual and haptic search, a target is easily found among distractors, this is called a pop-out effect. The target feature is then believed to be salient, and the search is performed in a parallel way. We investigated this effect with movable stimuli in a haptic search task. The task was to find a movable ball among anchored distractors or the other way round. Results show that reaction times were independent of the number of distractors if the movable ball was the target but increased with the number of items if the anchored ball was the target. Analysis of hand movements revealed a parallel search strategy, shorter movement paths, a higher average movement speed, and a narrower direction distribution with the movable target, as compared with a more detailed search for an anchored target. Taken together, these results show that a movable object pops out between anchored objects and this indicates that movability is a salient object feature. Vibratory signals resulting from the movable ball were found to be a reasonable explanation regarding the sensation responsible for the pop-out of movability.
\end{abstract}

Keywords Haptic search · Pop-out · Motion perception . Exploratory movements

Humans are able to recognize many common objects and discriminate them from others by just touching them. An everyday example of this is the search for your keys in a full bag. When trying to find the keys, one searches for certain characteristic features of the keys that set them apart from the other things in the bag-for example, the coldness

V. van Polanen $(\bowtie) \cdot$ W. M. Bergmann Tiest $\cdot$ A. M. L. Kappers Helmholtz Institute, Utrecht University,

Padualaan 8,

$3584 \mathrm{CH}$ Utrecht, The Netherlands

e-mail: V.vanPolanen@uu.nl or hardness of the metal. When objects are searched for, some searches are easier because certain features are more distinctive than other features and have the property to "stand out" from others. This phenomenon is called the pop-out effect. In this case, the target property that is to be found pops out from distractor items that do not exhibit the target property (Treisman \& Gelade, 1980). The pop-out effect has been found not only in vision (Treisman \& Gelade, 1980; Treisman \& Souther, 1985; Wolfe, 1998), but also in haptic search (Lederman \& Klatzky, 1997; Plaisier, Bergmann Tiest, \& Kappers, 2008, 2009). An easy visual example of this is the pop-out of a red dot among green dots. When the task is to find the red dot, it is immediately seen, without the need for a thorough search. In contrast, when a slanted line is searched for among upright lines, the search is more difficult. The slanted line is less distinctive from the upright line distractors.

Another interesting phenomenon is when the pop-out effect disappears when the properties of the target and distractor are interchanged; this is known as a search asymmetry. For example, the search is more efficient when a rough item is searched for than when a smooth item is the target (Plaisier et al., 2008). In other words, the rough item pops out from among the smooth items, but a smooth item does not pop out from among rough items.

In the situations described in the examples above, often the distinction between a parallel and a serial search strategy is made. In the case of a serial strategy, the search is more difficult, and all items must be explored one by one to find out whether it has the target property. In a parallel search, multiple items can be searched at once, which makes the search much easier. In an experiment, typically, reaction times are measured to illustrate the difference between these two search strategies. Reaction times are longer in the serial strategy and, because additional time is 
needed for each extra item, reaction time tends to increase with increases in the number of distractors. In the case of a parallel strategy, the reaction time is independent of the number of items, and the target pops out. If a feature pops out, it is believed to be a salient feature. In this way, the slope of reaction time against the number of items (the search slope) can be used as a tool to investigate whether a feature pops out and is salient.

However, the distinction between parallel and serial search based on the value of the slope appears not to be as strict as suggested above. In visual research, the search slope for a parallel search is predicted to be near zero, whereas a serial search has a positive slope (Treisman \& Souther, 1985). Yet a range of slopes has been found in visual search (Wolfe, 1998). In haptic search, a zero slope has rarely been found, and the analysis of hand movements offers a better perspective for the distinction between serial and parallel search (Plaisier et al., 2008). These authors found that a single hand-sweep was enough to detect a rough target among smooth distractors, whereas more detailed movements were needed in search for a smooth target. In addition, Plaisier, Kappers, Bergmann Tiest, and Ernst (2010) found a zero slope in a search that clearly showed serial hand movements. So it seems that the analysis of hand movements in active haptic search is important for the interpretation of the search slopes and can be used to investigate the saliency of object properties.

Salient features are important properties of objects that are most likely to be used to recognize an object and discriminate it from others. These features are easily perceived and are, therefore, more likely to be used in the early phases of object recognition (Lederman \& Klatzky, 1997). Learning more about the saliency of object properties might eventually lead to the identification of haptic primitives. Haptic primitives might be similar to visual primitives, which are defined as basic features that are automatically and preattentively extracted from a visual scene (Treisman \& Souther, 1985). In previous haptic studies, a pop-out effect has been found for roughness (Lederman \& Klatzky, 1997; Plaisier et al., 2008), coldness (Plaisier \& Kappers, 2010), edges and vertices (Plaisier et al., 2009), and surface contour (hole vs. no hole and slanted vs. flat; Lederman \& Klatzky, 1997), and these features can be considered salient in haptic search.

In the present study, we wanted to extend the research into feature saliency with an object property that has been little investigated in haptic research up to now-that is, the perception of motion. For the recognition of movable objects and, perhaps even more, for recognizing an object's function, perception of motion or movable parts is likely to be important. Chan, Whitaker, and Newell (2008) investigated the effect of object presentation in a task where participants haptically explored objects that consisted of specific pairs of shape and motion. Then they had to recognize the learned objects either by touch or by vision. Objects were more easily recognized in the test phase when they were presented as moving objects rather than static ones. Motion seemed to be an important property of the objects, because whereas shape information alone was sufficient to discriminate between the objects, participants performed better when motion was present.

Most studies investigating haptic perception of moving stimuli have been interested mainly in the sensitivity for direction (e.g., Evans \& Craig, 1991; Gleeson, Horschel, \& Provancher, 2009; Olausson, 1994; Placencia, Rahimi, \& Khoshnevis, 2009; Rinker \& Craig, 1994) or velocity (Salada, Colgate, Vishton, \& Frankel, 2004). Other research on haptic motion perception has focused on apparent motion (e.g., Harrar, Winter, \& Harris, 2008; Kirman, 1983). In apparent motion, two or more stimuli are presented sequentially at two different locations, but close enough and with a short time interval to create the illusory perception of a single moving stimulus. Apparent motion is a useful tool for investigating the perception of motion but might not be comparable to a natural situation. Research into the haptic perception of motion per se is limited. Provancher, Kuchenbecker, Niemeyer, and Cutkosky (2005) found, using a robotic device, that participants can distinguish between a rolling (movable) and an anchored object. However, in this study, a virtual object was used, and the finger was in a static position. We wanted to give participants a more active role in the perception of motion.

In this context, it is important to make the distinction between active and passive exploration. In passive exploration, stimuli are typically pressed to the fingers, whereas in active exploration, participants can freely explore the stimuli. One advantage of active exploration is that in addition to tactile information, kinaesthetic information is obtained. Shapes are recognized more accurately when they are actively explored, as compared with passive exploration (Heller, 1984). This illustrates the importance of active exploration in object recognition. Second, as was argued above, active exploration gives information about the saliency of object properties, whereas this is more limited in passive exploration (e.g., parallel passive search is limited to the number of fingers and, therefore, the set size and object size are limited). In order to fully perceive the motion of the object parts, an active exploration is needed. Despite this, many studies investigating saliency have used passive exploration and may, therefore, have underestimated the effect of kinaesthetic information. Also, in motion perception research, no study has involved active perceivers.

In short, no study has investigated the saliency of object motion and the detection of a movable item from among fixed items in a haptic search task. In visual search, an asymmetry has been found in perception between fast moving targets and slow targets (Ivry \& Cohen, 1992) and 
between moving and stationary targets (Royden, Wolfe, \& Klempen, 2001). Search was more efficient when the target was moving (or moving faster) and the distractors were stationary than the other way round. The question is whether, in haptic search, a search asymmetry also can be found. Since movability of dynamic parts in an object might be important for recognizing the object and, perhaps, its function, we hypothesized that movability is a salient feature.

To answer this question, we used a classical search task similar to that in Plaisier et al. (2008), in which participants had to determine whether a target was present among distractors. As target and distractors, movable and anchored items were used in two conditions. In the movable-target condition, participants had to search for a movable target among anchored distractors, and in the anchored-target condition, it was the other way round. Reaction times and hand movements were measured to examine which search condition was performed efficiently, in order to determine whether movability is a salient feature.

\section{Experiment 1}

\section{Method}

Participants Eleven participants took part in the experiment. Due to a large number of errors in one condition (more than $10 \%$ of the total number of trials in a condition), 1 participant was excluded from the analysis. The remaining 10 participants ( 3 males, 7 females) had a mean age of $21 \pm 3$ years and were all right-handed according to Coren's test (Coren, 1993). They used their dominant hand to perform the experiment. Participants gave informed consent and were paid for their participation.

Apparatus The stimulus set consisted of square laminated chipboard displays of $20 \times 20 \mathrm{~cm}$ in which holes had been drilled to fit 3, 5, 7, 9, or 11 items. The holes were located randomly on the display, and items on the display were at least $1 \mathrm{~cm}$ apart and at least $2 \mathrm{~cm}$ from the edge of the display, at the same locations as those used in Plaisier et al. (2008). The largest span of a configuration was $13 \mathrm{~cm}$ (measured from the center of the items), which was smaller than the hands of all the participants. There were two different displays for each possible number of items. The displays were also rotated $0^{\circ}, 90^{\circ}, 180^{\circ}$, and $270^{\circ}$ in the experiment, resulting in eight possible configurations for each item number. The items on the display consisted of ball transfer units (see Fig. 1) in which the ball either could move or was anchored. The anchored ball transfer units were produced by gluing the ball to its casing. Except for their ability to move, the items were indistinguishable. The diameter of a ball transfer unit was $31 \mathrm{~mm}$, with a ball $15 \mathrm{~mm}$ in diameter.

The experimental set-up is pictured in Fig. 2. The displays were presented on a table, to the left of a handrest. The handrest was level with the displays, so participants could easily slide from the rest to the display. A metal strip (15 $\mathrm{mm}$ wide) was fixed between the handrest and the stimulus display, to trigger the start of the measurement. When participants moved over the strip, a change in potential started the clock. The end of the measurement was induced by a verbal response from the participants, recorded with a microphone placed in front of the participants. The time difference between touching the strip and the response was the reaction time.

Hand movements were recorded using an NDI Optotrak Certus system. Two infra-red emitting diodes (IREDs) were placed on the right, dominant hand of participants: one on the nail of the index finger and one on the back of the hand near the wrist. Data were sampled with a frequency of $100 \mathrm{~Hz}$, resulting in 10-ms accuracy for reaction times and hand movement data. The spatial accuracy of the Optotrak was $0.2 \mathrm{~mm}$.

Task On each trial, the participant had to explore a single stimulus display, which was filled with a number of items (i.e., ball transfer units). The experiment consisted of two conditions. In the movable-target condition, all items were anchored if no target was present (target-absent trials), or one of the items was movable (target-present trials). In the anchored-target condition, all items were movable if no target was present (target-absent trials), or one item was anchored (target-present trials). Participants were asked to

Fig. 1 A ball transfer unit, right in cross-section. $\mathrm{a}=15 \mathrm{~mm}$, $\mathrm{b}=31 \mathrm{~mm}$
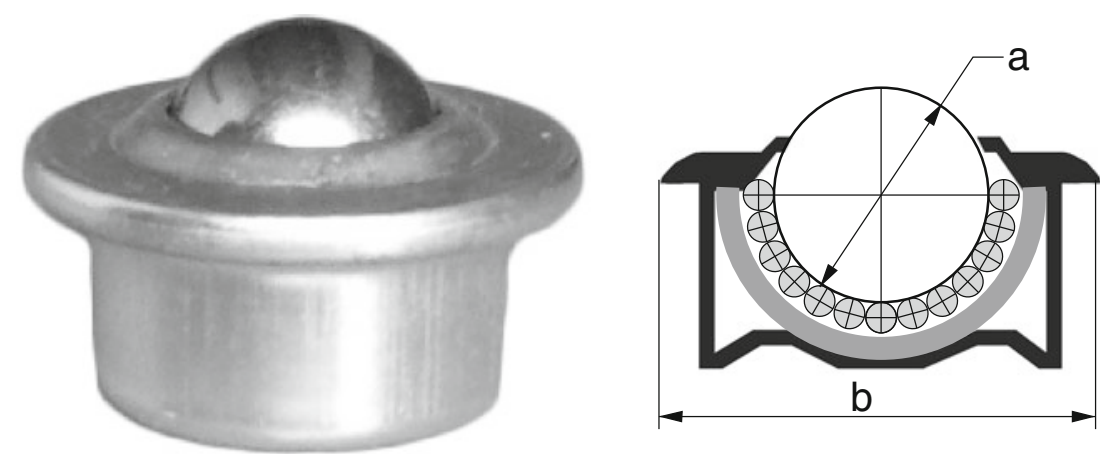


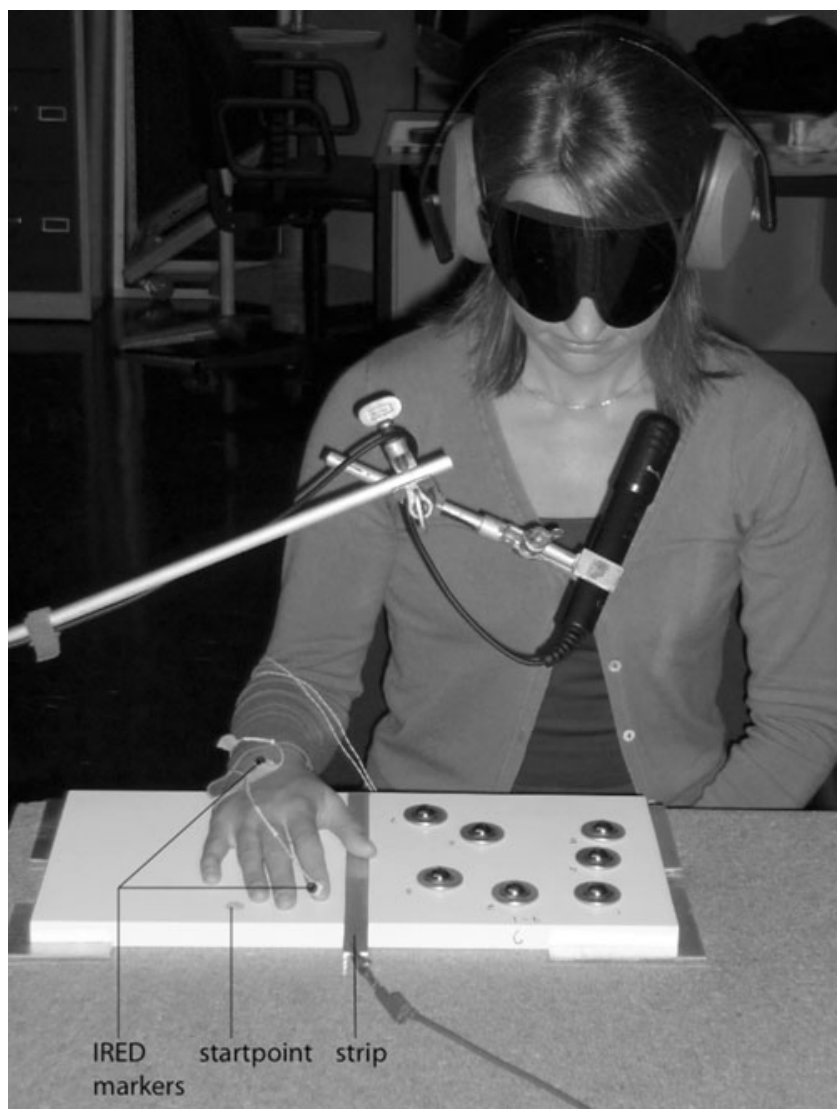

Fig. 2 Experimental setup. The participant just started the trial, moving over the metal strip. The IRED markers and starting point (bump) are indicated in the figure

move their hand across the display and had to respond whether a target was present or not by calling out the Dutch equivalents of "yes" and "no." They were instructed to determine as quickly as possible whether a target was present or not, but also to make as few mistakes as possible. They were told that they could move their hand over the display in the way they preferred.

Procedure First, the task and procedure were explained to the participants. Next, they put on a blindfold and were seated in front of the display. Participants wore hearing protectors and listened to white noise during the trials to block out the rolling noise and subsequent clues from the ball transfer units.

Before each trial, participants placed their hand on the handrest next to the display with their index finger on a start position, indicated by a small bump. The white noise was started to indicate that they could start their movement. Then they moved their hand over the display, starting on the righthand side. After they had responded verbally, the white noise was turned off, and they received feedback about their answer. Incorrect trials were repeated at the end of a session.

The order of the two search conditions was counterbalanced between participants. Each condition was divided into two sessions of approximately $45-60 \mathrm{~min}$. A session was preceded by a training session. At the first session of a condition, participants were told what to search for, and 15 practice trials were presented so that they could get comfortable with the nature of the task. During practice, the participants were encouraged to try out different strategies and to find a strategy in which they could perform the task as quickly and as accurately as possible. If necessary, more practice trials were presented until 9 out of 10 trials were answered correctly before the actual experiment began. For the second session of a condition, the same procedure was followed, except that a minimum of 10 trials was carried out until 9 out of 10 trials were correct.

The number of items was varied randomly. The location of the target was randomized among trials, and the displays were rotated in the experiment to obtain as many item locations as possible. In a condition, a target was never presented twice at the same location. In fact, on targetpresent trials, each configuration was unique. For each number of items $(3,5, \ldots, 11), 28$ trials were presented, resulting in a total of 140 trials per condition. On half of the trials, a target was present, and on the other half, the target was absent. The trials in a condition were divided over the two sessions in such a way that each number of items was presented equally often in a session (i.e., 14 trials per number of items, of which half contained a target in one session).

Analysis On incorrect trials, the reaction time could not be determined reliably, because the reaction time was measured as soon as the first response was given. For example, a very fast but wrong answer might just be a guess. Therefore, only correct trials were analyzed. Due to measurement errors, 6 trials $(0.2 \%)$ were excluded from the reaction time analysis, and 67 trials $(2 \%)$ from the Optotrak analysis. The measurement errors for the Optotrak mainly involved too large a number of missing data points, because the marker was not visible to the camera.

Mean reaction times were determined for each number of items in each condition and were plotted against the number of items for target-present and target-absent trials separately. A regression line was fitted through the data to determine the relation between reaction time and set size (i.e., the search slope). The slope and intercept of each regression line were calculated.

Missing values $(<1 \%)$ in the hand movement data were interpolated using a second-order polynomial function. Pilot data showed that the marker on the index finger usually crossed the metal strip first, so the start of a hand movement measurement was determined as the point at which the index finger passed the middle of the metal strip. The end of the hand movement was determined as many data points later as the reaction time for that trial, which corresponded to the time the participants gave a verbal response. Participants mainly 
moved their whole hand and did not spread their fingers, so the movement track of the finger marker was sufficient to describe the whole hand movement. Only data from this marker were used in the further analysis.

The total distance traveled and the average speed were calculated for each trial. The traveled distance (in millimeters) was the sum of the distances between each pair of successive data points in a movement track. The average speed (in millimeters/second) was simply the mean of the sum of distances multiplied by the sample frequency. The traveled distance and average speed were averaged to obtain a value for each condition, item number, and target-present and targetabsent trials separately. Regression lines were fitted on distance and speed data to investigate the change with number of items.

Furthermore, the distribution of movement directions was determined for each trial. The movement direction was calculated for each pair of sequential data points and grouped in bins of $10^{\circ}$. The number of movement directions in each bin was then counted. Next, the data were normalized by dividing by the total number of data points in a trial to obtain a relative measure of movement direction. The direction distributions were pooled over item number, because differences between numbers of items appeared to be small. To determine the spread of the direction distributions, the mean direction vector was calculated using circular statistics. The length of this vector was a measure for the uniformity of the distribution, with greater lengths indicating that movements were made mainly in a single direction.

Statistics For all variables, except the direction distributions, values that differed more than three standard deviations from the mean were removed. Because only the increase or decrease with number of items was of interest, separate 2 (condition) $\times 2$ (target presence) repeated measures analyses of variance (ANOVAs) were performed on the slopes of all the regression lines. The mean values of the reaction time, distance traveled, average speed, and direction distribution uniformity were collapsed over number of items and evaluated by four separate 2 (condition) $\times 2$ (target presence) repeated measures ANOVAs. Significance level was set to .05 , and post hoc tests were performed using paired-sample $t$-tests with a Bonferroni correction. Only interesting comparisons were made in the post hoc tests (e.g., differences in condition or target presence were examined, but not combinations of those factors). ANOVAs on the intercepts are not presented, because the same effects were found as in the analysis on the mean values. Only significant results will be presented.

Results

Errors Overall, participants made few errors (Table 1). More errors were made in the anchored-target condition, especially with a high number of items on the display.
Table 1 Proportion of errors for each condition of the total number of trials. "Yes/no" represents the proportion of self-corrected errors immediately after answering of the total number of trials

\begin{tabular}{|c|c|c|c|}
\hline & Items & Errors $(\%)$ & Yes/No $(\%)$ \\
\hline \multirow[t]{5}{*}{ Anchored target present } & 3 & 1 & 1 \\
\hline & 5 & 3 & 0 \\
\hline & 7 & 5 & 2 \\
\hline & 9 & 9 & 2 \\
\hline & 11 & 19 & 3 \\
\hline \multirow[t]{5}{*}{ Anchored target absent } & 3 & 1 & 1 \\
\hline & 5 & 1 & 1 \\
\hline & 7 & 1 & 1 \\
\hline & 9 & 0 & 0 \\
\hline & 11 & 1 & 1 \\
\hline \multirow[t]{5}{*}{ Movable target present } & 3 & 1 & 0 \\
\hline & 5 & 1 & 1 \\
\hline & 7 & 4 & 2 \\
\hline & 9 & 6 & 1 \\
\hline & 11 & 6 & 0 \\
\hline \multirow[t]{5}{*}{ Movable target absent } & 3 & 1 & 0 \\
\hline & 5 & 1 & 0 \\
\hline & 7 & 0 & 0 \\
\hline & 9 & 1 & 0 \\
\hline & 11 & 3 & 2 \\
\hline Total & & 4 & 1 \\
\hline
\end{tabular}

Note. Percentages were calculated on the basis of 14 trials + the number of errors (these were repeated) for each participant and subsequently averaged. Possible repeated measurement errors are not included

Table 1 shows that a substantial number of these errors could be classified as "yes/no" $(n=25,<1 \%$ of the total number of trials), in which participants corrected themselves immediately after answering. This was still considered a wrong answer but represents a separate case. Because of the small number of these errors, we did not expect that they would have an influence on the results. Furthermore, more false negative errors (i.e., participants missing a target that was present) were made than false positive errors (participants reporting a target that was not present).

Reaction times and search slopes The regression lines for the reaction time data for each condition are shown in Fig. 3, and slopes and intercepts in Table 2. As can clearly be seen, the search slopes are flatter for the movable-target condition (gray lines) than for the anchored-target condition (black lines). The ANOVA showed that searching for an anchored target resulted in higher slopes, as compared with the search for a movable target, $F(1,9)=41, p<.001$. All calculated slopes were significantly different from zero (all $p \mathrm{~s}<.01$ ).

The ANOVA on the reaction times demonstrated main effects of condition, $F(1,9)=132, p<.001$, and target 


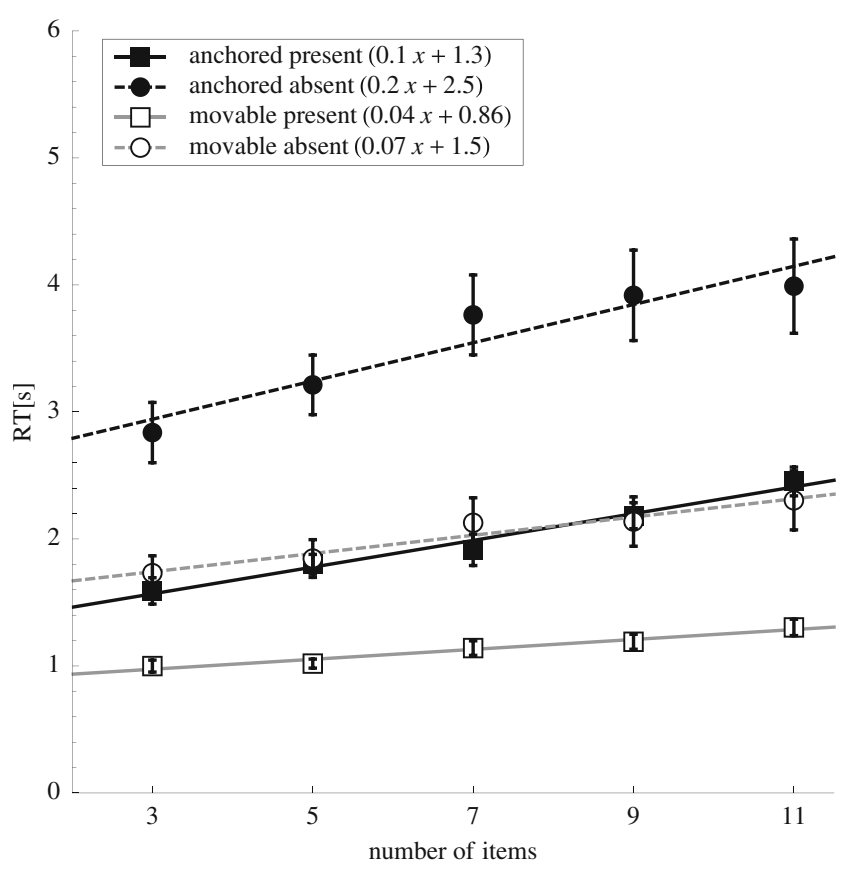

Fig. 3 Search slopes for both conditions, for target-present and absent trials separately. Slope values and intercepts are displayed in the legend above. Error bars represent standard errors of the means

presence, $F(1,9)=37, p<.001$. In the anchored-target condition, the reaction times were longer, as compared with the condition with a movable target. Participants were faster if a target was present than if a target was absent. Also, there was an interaction effect of condition and target presence, $F(1,9)=20, p=.002$. Post hoc tests revealed that all relevant comparisons were significant. The interaction effect indicated that the difference between the absence and presence of an anchored target was larger than the difference in the movable-target condition.

Table 2 Results of the regression analysis for reaction time (RT), traveled distance, and average speed. Upper rows represent the intercept, lower rows the slope. Values are shown for the anchoredtarget condition and the movable-target condition for target-present and target-absent trials separately

\begin{tabular}{lllll}
\hline & $\begin{array}{l}\text { Anchored } \\
\text { Present }\end{array}$ & $\begin{array}{l}\text { Movable } \\
\text { Present }\end{array}$ & $\begin{array}{l}\text { Anchored } \\
\text { Absent }\end{array}$ & $\begin{array}{l}\text { Movable } \\
\text { Absent }\end{array}$ \\
\hline RT $(\mathrm{s})$ & 1.3 & 0.86 & 2.5 & 1.5 \\
$(\mathrm{~s} /$ item $)$ & $0.1^{* *}$ & $0.04^{* *}$ & $0.2^{*}$ & $0.07^{* *}$ \\
Distance $(\mathrm{mm})$ & 228 & 292 & 604 & 517 \\
$(\mathrm{~mm} /$ item) & $14^{* *}$ & 2.1 & $16^{* *}$ & $7.0^{*}$ \\
Speed $(\mathrm{mm} / \mathrm{s})$ & 182 & 337 & 241 & 336 \\
$(\mathrm{~mm} / \mathrm{s} /$ item $)$ & -2 & $-7 * *$ & -4 & $-6^{*}$ \\
\hline
\end{tabular}

*Significant at .05 level

$* *$ Significant at .01 level

Note that for the intercepts, significance levels are not shown, but all intercepts had a $p<.01$.
Movement analysis Movement tracks of the finger showed different search strategies in the two conditions. Examples of movement tracks from 1 participant are shown in Fig. 4. On the left side, the anchored-target condition is illustrated. In this condition, items were searched in a complex pattern, especially when more items needed to be searched and a target was absent. In the movable-target condition, shown on the right side of Fig. 4, the movements were simpler. The participants moved more or less in a straight line over the display, whereas in the anchoredtarget condition, more detailed movements were necessary.

Additionally, quantitative variables demonstrated differences in the hand movements in the two search conditions. Results for the traveled distance are displayed in Fig. 5, and the average speed is shown in Fig. 6. Slopes and intercepts are indicated in Table 2. The ANOVA on the traveled distance revealed that the traveled distance was larger in the anchored-target condition than in the movable-target condition, $F(1,9)=19, p=.002$. However, an interaction effect of condition and target presence, $F(1,9)=27, p=.001$, showed that this difference was significant only if a target was absent. For both search conditions, the traveled distance was much shorter if a target was present than if it was absent, $F(1,9)=44, p<.001$.

The regression analysis on traveled distance revealed that there was a linear increase with the number of items on both target-present and target-absent trials for the anchored-target condition. A slope significantly different from zero was also found on the target-absent trials for the movable-target condition, but not for target-present trials. The slopes differed significantly between the conditions, $F(1,9)=33, p<.001$, with a larger increase with increases in the number of items for the anchored-target condition.

The average movement speed was higher when a movable target was searched for than when an anchored target was searched for, $F(1,9)=95, p<.001$. An effect of target presence, $F(1,9)=20, p=.002$, revealed a higher average speed on target-absent trials. However, there was also an interaction of condition and target presence, $F(1,9)=$ $7.9, p=.02$. Post hoc tests showed that this difference between absent and present trials was significant only in the anchored-target condition. In both conditions, the average speed decreased with the number of items, but only in the movable-target condition were the slopes significantly different from zero. An ANOVA demonstrated more negative slopes in the search for a movable target, $F(1,9)=9.9$, $p=.01$, but the interaction of condition and target presence, $F(1,9)=13, p=.005$, revealed that this was significant only on target-present trials. This interaction further showed that the slope was steeper on target-absent trials than on target-present trials when an anchored target was searched for.

The movement tracks in Fig. 4 suggest that in the movabletarget condition, side-to-side movements were dominant, 
Fig. 4 Examples of movement tracks of the finger marker made by 1 participant in the anchoredtarget condition (left) and the movable-target condition (right). Upper rows are target-present trials with the target indicated by a dark gray circle. Light gray circles represent distractor items. The black dot indicates the starting point of the track
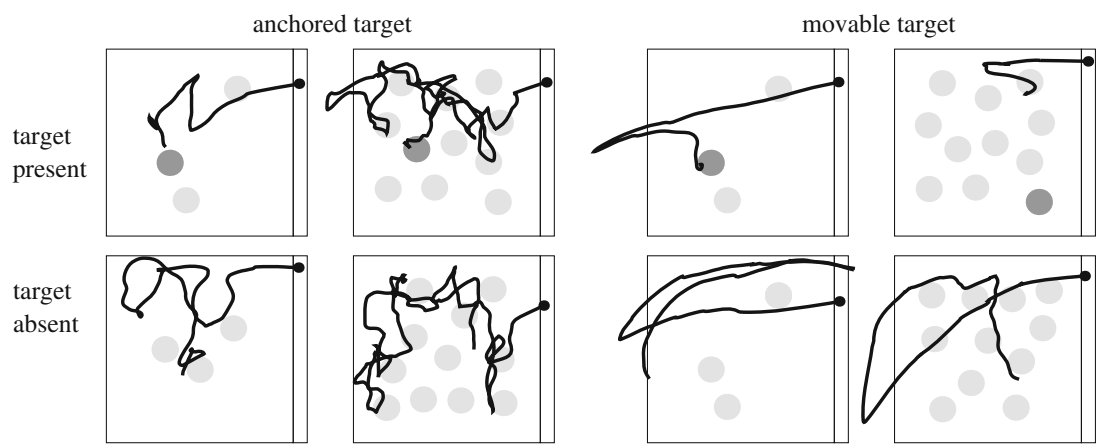

whereas in the anchored-target condition, participants needed to move in all directions. To test this, the distribution of movement directions was calculated, and the results are shown in Fig. 7. It can be seen that in the movable-target condition, there was a large component in the horizontal direction. In the case when a target was present, the main component was a movement to the left. The ANOVA on the length of the mean direction vector revealed an effect of target presence, $F(1,9)=155, p<.001$. This indicated that the distribution was more equally divided over all directions when no target was present. An effect of condition just failed to reach significance, $F(1,9)=4.8, p=.06$. Possibly, this was because the data resembled axial data, and opposite movement directions canceled out each other. That is why we calculated the length of the mean vector again after doubling the angles (Batschelet, 1981). An ANOVA demonstrated an effect of condition, $F(1,9)=12, p=.008$, which showed a more uniform distribution of movement directions in the anchored-target condition, as compared with the movable-target condition.

\section{Discussion}

In the search for a movable target, the search slopes were low, and reaction times were independent of the number of items. Searching for an anchored target showed large reaction times that increased with more distractors in the display. This indicates that the search for a movable target among anchored distractors is easier than the reversed situation. In addition, hand movement analysis revealed a difference in search strategy between the two tasks. A shorter traveled distance, a high average speed, and movements mainly in the horizontal direction suggest the use of a parallel strategy when a movable target is searched for.

Taken together, the results demonstrate a pop-out effect for movable stimuli. A point of discussion is to what extent movability can be considered a feature or object property. In other words, what is the feature that makes movability pop out, and how is it perceived? It is important to note that in this study, the items moved upon touch, so the movable item was more stationary relative to the skin of the finger than was the anchored item. The question is what sensation was responsible for the "movability" detected in the task. A few possible sensations might be perceived in order to feel "movability."

First, the mechanical interactions between the movable ball and the small ball bearings in the casing might produce small vibratory sensations or small forces that are perceived by the hand. This creates the feeling of an object that moves with respect to another object. There is always some amount of friction between the two objects, and this can be detected. In the anchored items, these signals are absent and, thus, might be the discriminating feature between the two stimuli.

Second, when one moves over a ball transfer unit with a constant force applied to the display, the reaction force changes as the skin touches a rolling ball because the ball moves along with the hand. Over time, the movement against the skin, the velocity, and the reaction force differ. This variability is not present in a stationary ball, which
Fig. 5 Traveled distance for the anchored-target condition (left) and the movable-target condition (right). Light gray bars represent target-present trials, and dark gray bars target-absent trials. Error bars indicate standard errors of the means
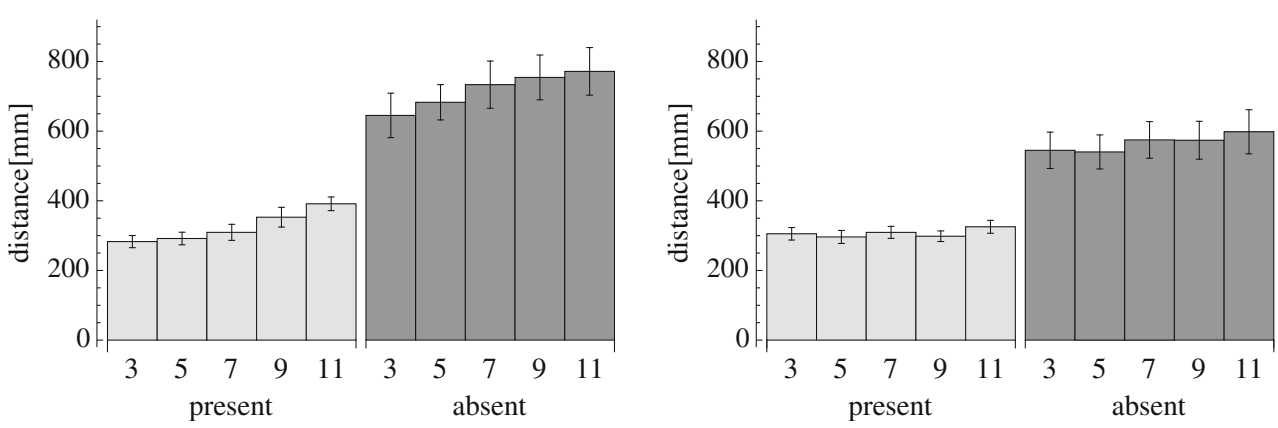
Fig. 6 Average speed for the anchored-target condition (left) and movable-target condition (right). Light gray bars represent target-present trials, and dark gray bars target-absent trials. Error bars indicate standard errors of the means

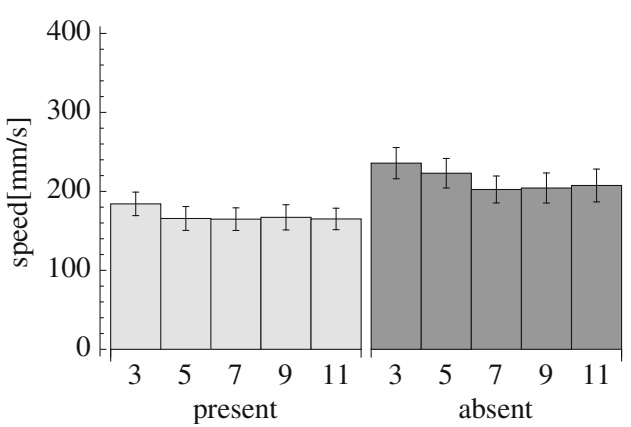

applies a constant reaction force upon pressure. Receptors in the skin might be more sensitive to these changes in force or velocity, resulting in the pop-out of movable items. In a study of Smith, Gosselin, and Houde (2002), participants kept a constant normal force in a search task. Possibly, they did this to feel the changes in tangential force that accompany the touch of a target. Moreover, the variability in reaction force might be present not only in time, but also in location. The ball moves with respect to its casing, so there is a difference in perception triggered by the moving ball and the stationary casing or the stationary distractors that touch other parts of the hand.

Lastly, instead of perceiving the actual force, the amount of skin stretch is also susceptible to changes in reaction force, which can be noticed by the skin receptors. The movable ball results in less stretch of the skin than does the anchored ball. Again, this could be a variation in either time or location. The amount of skin stretch resulting from the movable item deviates with respect to the anchored items and the stationary background. These differences in stretch of the skin might possibly be the distinctive perception that accompanies the touch of a movable object.

We wanted to further investigate possible explanations for the pop-out of movable stimuli. Therefore, we set up an experiment to explore some physical properties of the stimulus display. According to the discussion above, three possible sensations could be considered: mechanical interactions, reaction force, and skin stretch. Mechanical interactions might lead to small forces and vibrations that might be perceived by the participants. Following this reasoning, we measured the amount of vibrations of the whole stimulus (i.e., the display filled with ball transfer units) in Experiment 2. The reaction force and skin stretch were evaluated by measuring the amount of friction of the stimulus.

If differences in friction or vibration are found between target-present trials and target-absent trials, participants could have used this variable as a cue to find the target. Moreover, if a change in a variable is found in the presence of a movable target, as compared with the case when a
Fig. 7 Movement direction distribution for each condition, target-present and target-absent trials separately. The radial axis represents the proportion of movements made in a direction bin

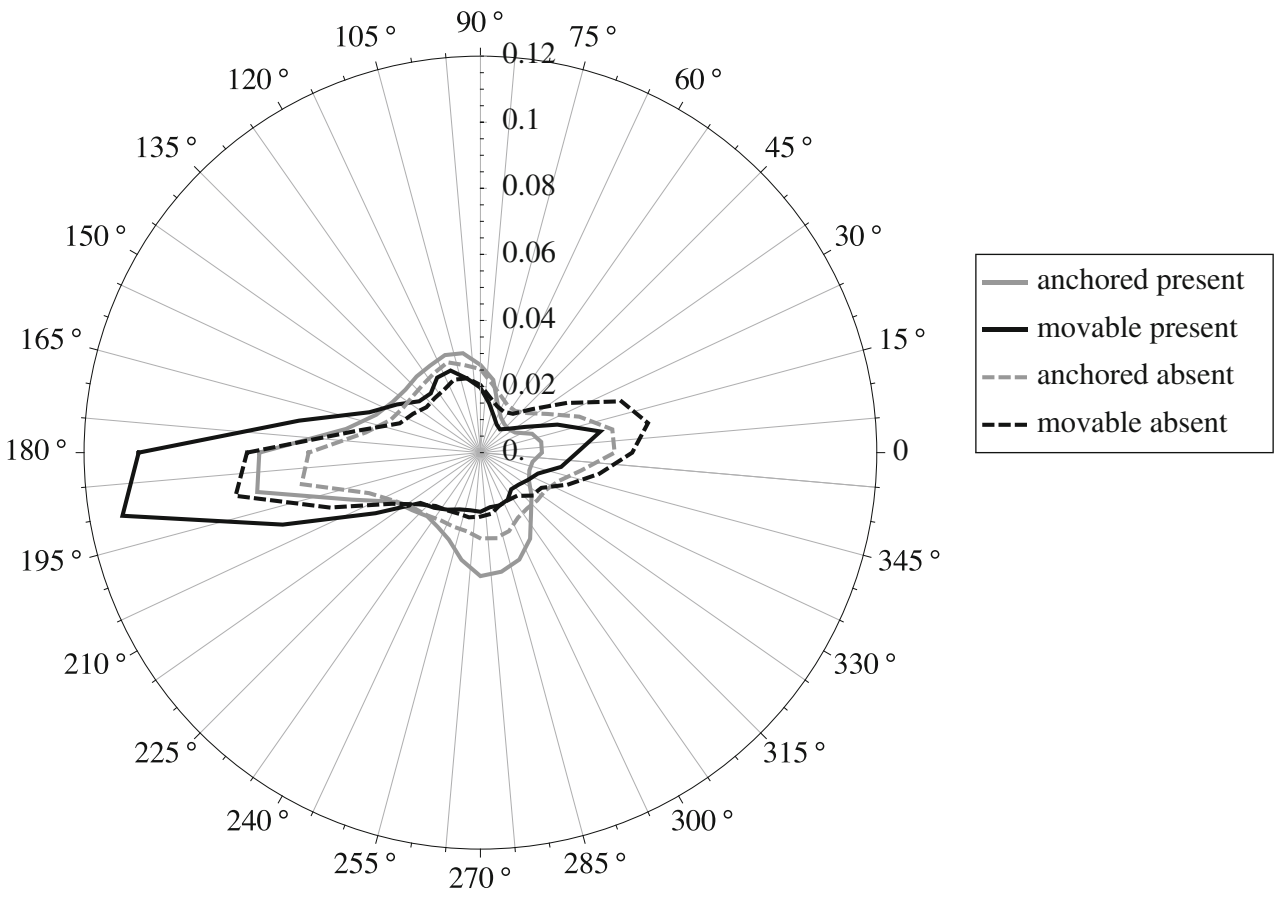


movable target is absent, this could explain how participants detect movability. If this change is relatively larger in the movable-target condition than in the anchored-target condition, this would provide a possible explanation for the search asymmetry found in Experiment 1.

\section{Experiment 2}

\section{Method}

Apparatus The experimental setup is shown in Fig. 8. A force-moment sensor (Nitta, IFS-67M25A25-I40) was placed underneath the stimulus display, which was filled with seven ball transfer units. A $20 \times 20 \mathrm{~cm}$ board, the same size as the stimulus display, was pulled $6 \mathrm{~cm}$ over the display (from $3 \mathrm{~cm}$ to the right of the edge to $3 \mathrm{~cm}$ to the left). A weight was placed on the board, making the total mass $1,009 \mathrm{~g}$ (about $10 \mathrm{~N}$ ). The board was pulled by two strings that were attached to a lateral translator (Isel C142-1), which moved at a constant speed of 10 or $40 \mathrm{~mm} / \mathrm{s}$. The speeds were much lower than the speeds used by participants in Experiment 1 but were chosen, along with the short distance, to keep the setup stable and avoid tipping of the board. The sample frequency of the force sensor was $1000 \mathrm{~Hz}$.

The board was covered with a thin layer of leather chamois in order to resemble the hand's skin. This was done for two reasons. The first reason was to increase the overall amount of friction and improve the signal-to-noise ratio. Second, the ball transfer units appeared to be slightly different in height. By using the chamois, the material adapted itself to the ball height, and in this way, all balls were touched and could contribute to the measurement. The board was not exactly like the human hand, because the intention of the experiment was not to simulate the human hand, but to give insight into the physical properties of the stimulus that might be perceived by participants.

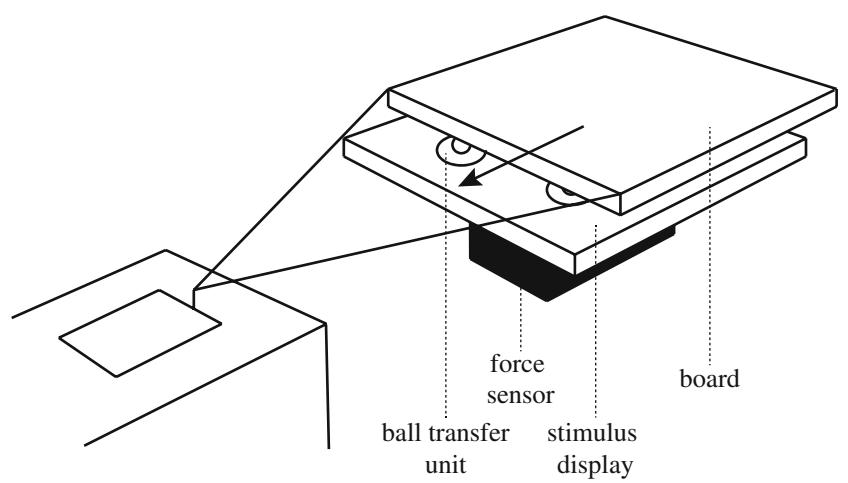

Fig. 8 Setup of Experiment 2. A board covered with leather chamois is pulled over the stimulus display, which is placed upon a force sensor
Procedure Four sets of measurements were performed for each speed level: two in the movable-target and two in the anchored-target conditions. In the movable-target condition, a movable target could be absent or present among anchored distractors. In the anchored-target condition, an anchored target was present or absent among movable distractors. A single configuration of seven items was used for all measurements, so in the measurement sets mentioned above, there were zero, one, six, or seven movable items, respectively. For the low speed, 12 trials were measured for each situation, and three different target locations were used. Eight trials were measured for each situation with a high speed, with two target positions. ${ }^{1}$

Analysis From the raw data, only the forces in the direction of movement were analyzed. The middle part of $4 \mathrm{~cm}$ was extracted to exclude forces due to the starting and stopping of the motor. Of this part, the mean force was calculated. Also, the data were high-pass filtered using a second-order bidirectional Butterworth filter with a cutoff frequency of $10 \mathrm{~Hz}$ to eliminate the offset and slow changes. Of these filtered data, the power spectral density (psd) was calculated using Welch's method. A window of $500 \mathrm{~ms}$ was used, with an overlap of $50 \%$. Then the psd was integrated to get an estimate of the overall vibration. Mean force and the integrated psd were averaged over trials.

\section{Results and discussion}

An example of the force and psd of a trial are shown in Fig. 9. Mean results are displayed in Fig. 10. The amounts of friction and vibration are plotted against the number of movable balls for the two speeds. Regression lines were fitted to the data, showing the dependence of the variables on the number of movable items. The fit was quite good for both speeds $\left(R^{2} \mathrm{~s}>.98\right)$. The mean force decreased with the number of movable balls, while the integrated psd increased. The interesting values are the differences within a condition between trials with and without a target. The absolute and relative differences can be found in Table 3.

The presence of an anchored target resulted in a relatively large increase in friction, as compared with when it was absent. In contrast, the relative change in the movable-target condition was quite small. When the change in friction between the conditions is compared, it would be expected that finding an anchored target is easier, since differences in this condition are larger. However, Experiment 1 showed that this was actually a more demanding task. Thus, it seems unlikely that friction

\footnotetext{
${ }^{1}$ Due to the limited availability of the force sensor and clearness of the results, we did not extend this number of trials for the high-speed trials.
} 


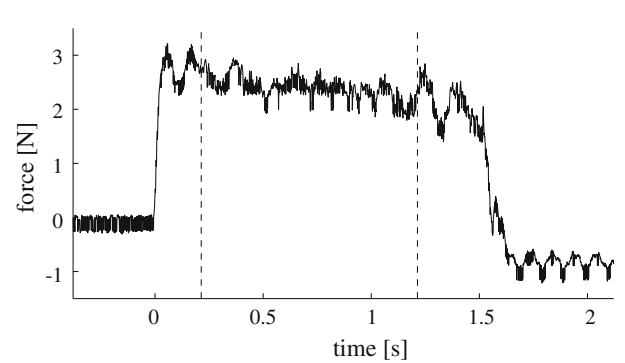

Fig. 9 Example of the force in the direction of motion (left) and the power spectral density (psd) after filtering (right) of a trial in the movable-target-present condition, measured at a high speed. In the

was a beneficial cue for participants to find a movable target, and it cannot explain the pop-out of movability.

The absolute differences in vibration were similar in the movable- and the anchored-target conditions, but there were differences in the relative differences. There was more vibration when a movable target was present than when the target was absent. So, the amount of vibration could be an explanation for the psychophysical results found in Experiment 1. Participants could feel an increase in vibration, which indicated that a movable target was present and that the display was not completely filled with distractors. Changes in the amount of vibration were especially large on the high-speed trials. Since participants moved at a much higher speed, perhaps they perceived an even larger difference. Because the relative difference between a movable target and no movable target was larger than the presence versus absence of an anchored target, the former task would be expected to be easier than the latter. This corresponds to the search asymmetry found in Experiment 1. Therefore, it is likely that participants used this vibration, resulting from the movement of the ball, as a cue to perform the search task.

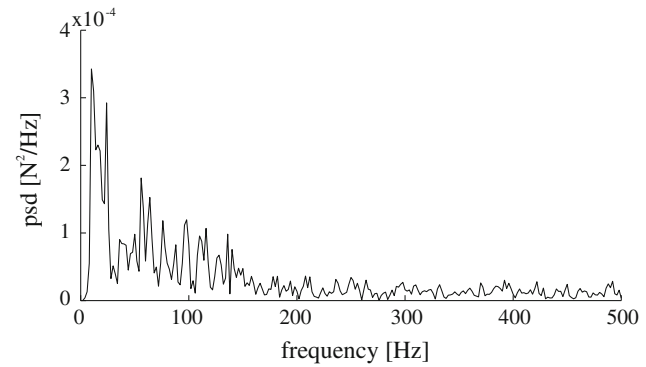

force plot, time is set at $0 \mathrm{~s}$ at the point the board starts moving. Dashed lines indicate the analyzed part of the data

\section{General discussion}

The aim of this study was to find out whether movability is a haptic salient feature. To investigate this, we used a haptic search task in which participants had to actively search for an anchored target among movable distractors or a movable target among anchored items. Reaction times and hand movements were measured to determine the search strategy and the saliency of the target feature. In short, the results show a search asymmetry between the two conditions. Differences in reaction times, in search slopes and in hand movements were observed between searching for an anchored target and finding a movable target. In the next paragraphs, these findings will be further discussed.

First, the results show shorter reaction times in the search for a movable target, as compared with searching for an anchored target. More important, there were large differences between the search slopes (i.e., the change in reaction time with the number of items). The slope value was much smaller in the movable-target condition than in the anchored-target condition. These search slopes indicate a search asymmetry. Searching for a movable target among
Fig. 10 Results of mean values plotted against the number of movable items $(0=$ movable absent, 1 = movable present, $6=$ anchored present, $7=$ anchored absent) for the amount of vibration (left) and friction (right). Error bars represent standard errors of the means
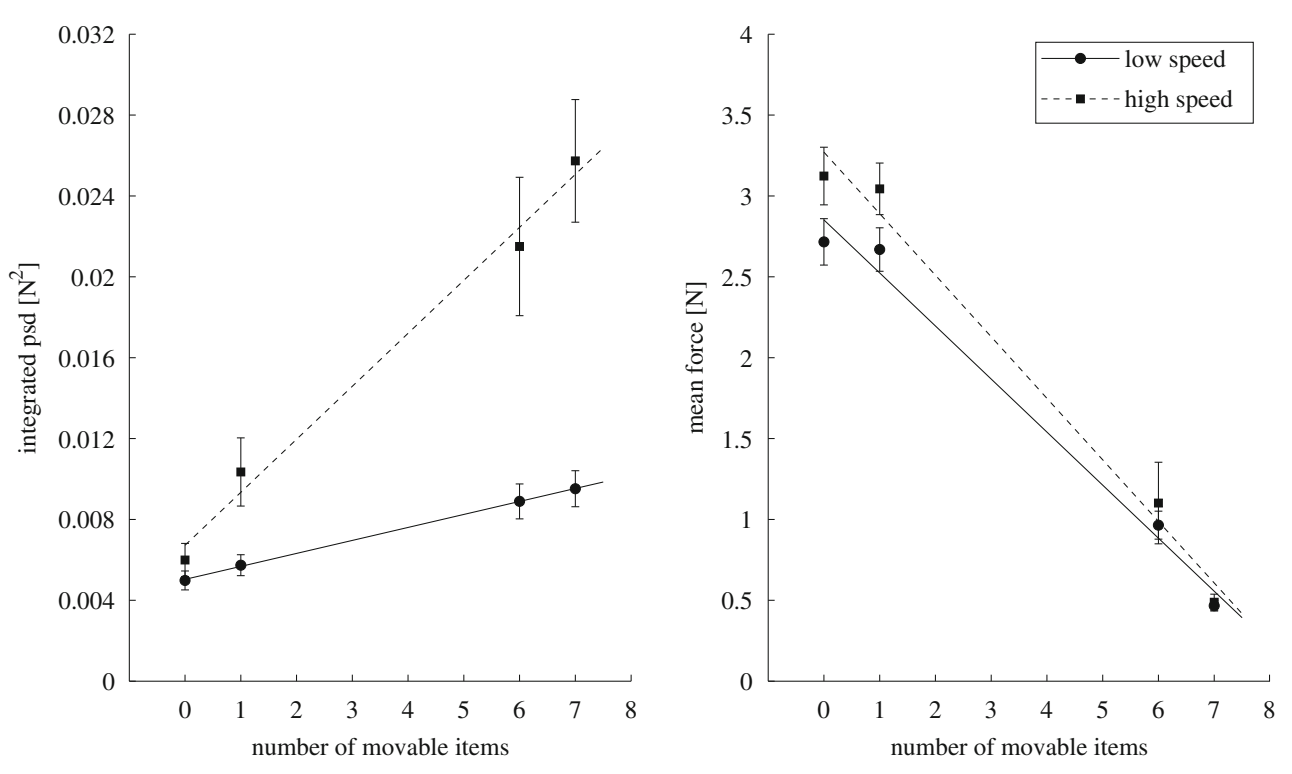
Table 3 Absolute and relative differences in friction and vibration between the presence and absence of a target. Relative differences were calculated by dividing the absolute difference by the targetabsent value

\begin{tabular}{|c|c|c|c|c|c|}
\hline & & \multicolumn{2}{|c|}{ Absolute Difference } & \multicolumn{2}{|c|}{ Relative Difference } \\
\hline & & Movable & Anchored & Movable & Anchored \\
\hline \multirow{2}{*}{$\begin{array}{l}\text { Low } \\
\text { speed }\end{array}$} & mean force $(\mathrm{N})$ & 0.047 & 0.50 & $2 \%$ & $107 \%$ \\
\hline & integrated psd $\left(\mathrm{N}^{2}\right)$ & $0.76 * 10^{-3}$ & $0.63 * 10^{-3}$ & $15 \%$ & $7 \%$ \\
\hline \multirow{2}{*}{$\begin{array}{l}\text { High } \\
\text { speed }\end{array}$} & mean force $(\mathrm{N})$ & 0.080 & 0.61 & $3 \%$ & $126 \%$ \\
\hline & integrated psd $\left(\mathrm{N}^{2}\right)$ & 0.0044 & 0.0042 & $73 \%$ & $16 \%$ \\
\hline
\end{tabular}

anchored distractors is relatively easy, but if target and distractor identity are interchanged, the search becomes considerably more difficult. If participants had to search for a movable target among anchored ones, search slopes were as low as $39 \mathrm{~ms}$ per item if a target was present. This is comparable to the results of previous studies that showed a pop-out effect when roughness $(20 \mathrm{~ms} / \mathrm{item}$; Plaisier et al., 2008), 3-D shape (25 ms/item; Plaisier et al., 2009), and coldness (32 ms/item; Plaisier \& Kappers, 2010) were investigated using tasks similar to that in this study.

As was argued in the introduction, hand movement analysis is important for the interpretation of the search slopes. The search slopes point to a difference in search strategy, and this is reflected in the hand movements of participants. As is illustrated in Fig. 4, participants did not need to move extensively over the whole display in the movable-target condition. Often, only one or two sweeps over the display were enough. The whole hand could be used to tell the presence of a target. In the anchored-target condition, this was not the case, and a more detailed exploration in all directions was necessary. Quantitative data confirmed this difference in movement strategy between the two conditions. Shorter traveled distances were found in the movable-target condition. As for reaction time, the traveled distance increased more with increases in the number of items in the search for an anchored target. When the values for the target-present trials were looked at, a slope of about $14 \mathrm{~mm}$ per item was found in the anchored-target condition, whereas this was only $2.1 \mathrm{~mm}$ per item in the movable-target condition. Additionally, participants moved at a lower speed in the anchored-target condition than in the movable-target condition. The detailed and slow movements in the anchored-target condition indicate a serial search strategy, whereas the fast and short movements in the movable-target condition point to a parallel strategy. Thus, the search asymmetry is also present in the hand movement data. The finding of a parallel strategy in the search for a movable target supports the claim for a pop-out effect of movability.
The movement strategies, combined with the low search slopes found for the movable-target condition, strongly suggest an efficient search strategy for this condition. Therefore, we conclude that a movable target pops out from among anchored distractors. Although the slope was significantly different from zero, the low slope value, in combination with the parallel search strategy, is enough to justify the conclusion of the presence of a pop-out effect, especially in relation to the anchored-target condition. Almost all participants reported that they found the search for an anchored target more difficult, and also the number of erroneous answers in the anchored-target condition indicates that this condition was more demanding. Taken together, it can be concluded that the search for a movable target among anchored distractors is easier than the other way round and that movability is a salient feature.

To get a better understanding of the nature of this popout of movability, we explored the possible underlying sensations responsible for this effect and the search asymmetry found. Mechanoreceptors in the skin can detect motion by spatial integration of pressure and by stretch of the skin (Olausson, Wessberg, \& Kakuda, 2000), but they are also sensitive to vibration (Johnson, 2001). As was discussed above, three possible sensations that could indicate the presence of movability come to mind: reaction force, skin stretch, and vibration.

To start with, there is change in perception of the reaction force when one moves over a rolling unit. Similarly, when one touches a rolling ball, the amount of skin stretch changes. In Experiment 2, we examined these possible explanations of the pop-out of movability in more detail. In particular, we investigated the amount of friction (reaction force and skin stretch) of the stimulus display in the different conditions with a varying amount of movable items. The presence of an anchored ball, as compared with its absence, resulted in a large relative increase in friction, while the relative change in the movable-target condition was much smaller. According to this large relative difference in friction in the anchoredtarget condition, one would expect the anchored ball to be easier to find. However, the psychophysical data showed that participants still found it hard to detect such a target. Although the friction change was high in the anchoredtarget condition, apparently participants could not use the friction cue optimally to perform the task. Because of the small differences in friction between the presence and absence of a movable target, it is unlikely that participants used the amount of friction to perform the search task. Therefore, friction cannot explain the pop-out of movability.

A third possible feature that could be perceived is the vibration produced by the mechanical interaction between the movable ball and the ball bearings. The results of Experiment 2 demonstrated more vibration in the presence of a movable target, as compared with the absence of the target. Thus, vibratory signals that are produced when the ball of the ball 
transfer unit is moved could be used as a cue to determine whether a movable target was present. Since adding an anchored target to a group of movable distractors resulted in a smaller relative difference, it would be expected that this search would be more difficult. This corresponds to the performance of the participants, who found it easier to detect a movable stimulus. Hence, vibration provides a reasonable explanation for the pop-out of movability.

A study by Whang, Burton, and Shulman (1991) supports the notion of a sensitivity for vibration amplitude change. In a cuing task, participants had to detect a change in vibration amplitude among constant amplitude distractors, or the other way round. Cuing improved performance more in the latter task than in the former. Detecting an amplitude change among constant amplitudes is easier, and this might indicate that vibration amplitude change pops out. In the present study, we propose a pop-out effect of movability, which can be detected by using the vibratory signals produced by the moving ball as a cue. It remains to be seen whether movability itself, without a vibrational cue, would still pop out.

In conclusion, the results of this study show that movable stimuli are perceived irrespective of the number of distractors and are searched with a parallel strategy. Therefore, movability is a salient feature. The vibratory signals produced by the movable object seem to be a reasonable explanation regarding the sensation that is responsible for the pop-out of movability. The perception of movability might be important for the exploration and recognition of dynamic stimuli. Furthermore, at a small scale, movability perception might play a role in the detection of (micro) slip and, hence, be important for the holding and handling of objects.

Author note This work was supported by the European Commission with the Collaborative Project 248587, "THE Hand Embodied", within the FP7-ICT-2009-4-2-1 program "Cognitive Systems and Robotics." The authors would like to thank Pieter Schiphorst for technical support and Yoshihiro Tanaka for his help in Experiment 2.

Open Access This article is distributed under the terms of the Creative Commons Attribution Noncommercial License which permits any noncommercial use, distribution, and reproduction in any medium, provided the original author(s) and source are credited.

\section{References}

Batschelet, E. (1981). Circular statistics in biology. New York: Academic Press.

Chan, J. S., Whitaker, T. A., \& Newell, F. N. (2008). Investigating visuo-tactile recognition of unfamiliar moving objects. Lecture Notes in Computer Science, 5024, 308-312.

Coren, S. (1993). The left-hander syndrome: The causes and consequences of left-handedness. New York: Vintage Books.

Evans, P. M., \& Craig, J. C. (1991). Tactile attention and the perception of moving tactile stimuli. Perception \& Psychophysics, 49, 355-364.
Gleeson, B. T., Horschel, S. K., \& Provancher, W. R. (2009). Communication of direction through lateral skin stretch at the fingertip. In Third Joint EuroHaptics Conference and Symposium on Haptic Interfaces for Virtual Environment and Teleoperator Systems (pp. 172-177). Los Alamitos, CA: IEEE Computer Society Press.

Harrar, V., Winter, R., \& Harris, L. R. (2008). Visuotactile apparent motion. Perception \& Psychophysics, 70, 807-817.

Heller, M. A. (1984). Active and passive touch: The influence of exploration time on form recognition. The Journal of General Psychology, 110, 243-249.

Ivry, R. B., \& Cohen, A. (1992). Asymmetry in visual-search for targets defined by differences in movement speed. Journal of Experimental Psychology. Human Perception and Performance, 18, 1045-1057.

Johnson, K. O. (2001). The roles and functions of cutaneous mechanoreceptors. Current Opinion in Neurobiology, 11, 455-461.

Kirman, J. H. (1983). Tactile apparent movement: The effects of shape and type of motion. Perception \& Psychophysics, 34, 96-102.

Lederman, S. J., \& Klatzky, R. L. (1997). Relative availability of surface and object properties during early haptic processing. Journal of Experimental Psychology. Human Perception and Performance, 23, 1680-1707.

Olausson, H. (1994). The influence of spatial summation on human tactile directional sensibility. Somatosensory \& Motor Research, 11, 305-310.

Olausson, H., Wessberg, J., \& Kakuda, N. (2000). Tactile directional sensibility: Peripheral neural mechanisms in man. Brain Research, 866, 178-187.

Placencia, G., Rahimi, M., \& Khoshnevis, B. (2009). Sensing directionality in tangential haptic stimulation. Lecture Notes in Artificial Intelligence, 5639, 253-261.

Plaisier, M. A., Bergmann Tiest, W. M., \& Kappers, A. M. L. (2008). Haptic pop-out in a hand sweep. Acta Psychologica, 128, 368-377.

Plaisier, M. A., Bergmann Tiest, W. M., \& Kappers, A. M. L. (2009). Salient features in 3-D haptic shape perception. Attention, Perception, \& Psychophysics, 71, 421-430.

Plaisier, M. A., \& Kappers, A. M. L. (2010). Cold objects pop out! Lecture Notes in Computer Science, 6192, 219-224.

Plaisier, M. A., Kappers, A. M. L., Bergmann Tiest, W. M., \& Ernst, M. O. (2010). Visually guided haptic search. IEEE Transactions on Haptics, 3, 63-72.

Provancher, W. R., Kuchenbecker, K. J., Niemeyer, G., \& Cutkosky, M. R. (2005). Perception of curvature and object motion via contact location feedback. Robotics Research, 15, 456-465.

Rinker, M. A., \& Craig, J. C. (1994). The effect of spatial orientation on the perception of moving tactile stimuli. Perception \& Psychophysics, 56, 356-362.

Royden, C. S., Wolfe, J. M., \& Klempen, N. (2001). Visual search asymmetries in motion and optic flow fields. Perception \& Psychophysics, 63, 436-444.

Salada, M., Colgate, J. E., Vishton, P., \& Frankel, E. (2004). Two experiments on the perception of slip at the fingertip. In Proceedings of the 12th International Symposium on Haptic Interfaces for Virtual Environment and Teleoperator Systems, (pp. 146-153). Chicago, IL.

Smith, A. M., Gosselin, G. V., \& Houde, B. (2002). Deployment of fingertip forces in tactile exploration. Experimental Brain Research, 147, 209-218.

Treisman, A., \& Gelade, G. (1980). Feature-integration theory of attention. Cognitive Psychology, 12, 97-136.

Treisman, A., \& Souther, J. (1985). Search asymmetry: A diagnostic for preattentive processing of separable features. Journal of Experimental Psychology. General, 114, 285-310.

Whang, K. C., Burton, H., \& Shulman, G. L. (1991). Selective attention in vibrotactile tasks: Detecting the presence and absence of amplitude change. Perception \& Psychophysics, 50, 157-165.

Wolfe, J. M. (1998). What can 1 million trials tell us about visual search? Psychological Science, 9, 33-39. 